INPLASY

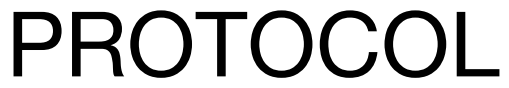

To cite: Che et al. Efficacy and safety of Guhong injection for coronary heart disease: $A$ protocol for systematic review and meta-analysis. Inplasy protocol 2021120032. doi: 10.37766/inplasy2021.12.0032

Received: 05 December 2021

Published: 05 December 2021

Corresponding author:

Sihua Che

294347339@qq.com

Author Affiliation:

Changchun University of

Chinese Medicine.

Support: Technology Project of Jilin.

Review Stage at time of this submission: The review has not yet started.

Conflicts of interest:

None declared.

\section{Efficacy and safety of Guhong injection for coronary heart disease: A protocol for systematic review and meta-analysis}

Che, S1; Wang, M²; Liu, L3; Zhang, S4; Shi, G55.

Review question / Objective: The aim of this meta-analysis of randomized controlled trials is to evaluate the effectiveness and safety of Guhong injection for the treatment of coronary heart disease.

Condition being studied: Coronary heart disease (CHD) is mainly the pathological change of coronary atherosclerotic plaque, which results in lumen stenosis or spasm, and eventually leads to myocardial ischemia, hypoxia, and even necrosis. Various risk factors are closely associated with the occurrence of CHD, such as age, smoking, hypertension, diabetes, hyperlipidemia, etc. CHD is considered one of the major causes of morbidity and mortality worldwide, posing a serious threat to public health. Current therapeutic approaches for CHD mainly focus on drug therapy and percutaneous coronary intervention( $\mathrm{PCl})$.However,there still exist some problems including drug side effects, adverse cardiac events after PCI(i.e. restenosis, stent thrombosis, and ischemia-reperfusion injury).

INPLASY registration number: This protocol was registered with the International Platform of Registered Systematic Review and Meta-Analysis Protocols (INPLASY) on 05 December 2021 and was last updated on 05 December 2021 (registration number INPLASY2021120032).

\section{INTRODUCTION}

Review question / Objective: The aim of this meta-analysis of randomized controlled trials is to evaluate the effectiveness and safety of Guhong injection for the treatment of coronary heart disease.
Condition being studied: Coronary heart disease (CHD) is mainly the pathological change of coronary atherosclerotic plaque, which results in lumen stenosis or spasm, and eventually leads to myocardial ischemia, hypoxia, and even necrosis. Various risk factors are closely associated with the occurrence of CHD, such as age, smoking, hypertension, diabetes, 
hyperlipidemia, etc. CHD is considered one of the major causes of morbidity and mortality worldwide, posing a serious threat to public health. Current therapeutic approaches for CHD mainly focus on drug therapy and percutaneous coronary intervention( $\mathrm{PCl})$. However,there still exist some problems including drug side effects, adverse cardiac events after $\mathrm{PCI}(i . e$. restenosis, stent thrombosis, and ischemia-reperfusion injury).

\section{METHODS}

Search strategy: We will perform a comprehensive systematic literature search in the following electronic databases: EMBASE, PubMed, Cochrane Library, Web of Science, China National Knowledgelnfrastructure (CNKI), WanFang Database, Chinese Biomedical Database (CBM), Chinese Scientific Journal Database (VIP). The retrieval time was from their inception to November 30, 2021. Using the following search terms: "Guhong injection", "coronary heart disease", "angina pectoris", and "randomized controlled trials". Illustrated by the case of PubMed, the detailed search strategy is shown in Table 1, which will make appropriate adjustments according to the specific database. Moreover, references of retrieved articles were also manually retrieved to obtain additional relevant studies.

Participant or population: The patients who meet the diagnostic criteria of CHD will be included, including stable angina pectoris (SAP) and unstable angina (UA). There are no restrictions on sex, age, andrace.

Intervention: The treatment group was treated with Guhong injection combined with conventional treatment.

Comparator: The intervention method of the control group was conventional treatment, such as b-blockers, clopidogrel, statins, aspirin, etc.

Study designs to be included: The study will incorporate all randomized controlled trials that evaluate the efficacy of Guhong injection for the treatment of CHD, with no limit to language, publication, time, or blinding.

Eligibility criteria: 1.Type of studyThe study will incorporate all randomized controlled trials (RCTs) that evaluate the efficacy of Guhong injection for the treatment of CHD, with no limit to language, publication, time, or blinding.2.Type of participant The patients who meet the diagnostic criteria of CHD will be included, including stable angina pectoris (SAP) and unstable angina (UA). There are no restrictions on sex, age, and race. 3.InterventionThe intervention method of the control group was conventional treatment, such as bblockers, clopidogrel, statins, aspirin, etc. The treatment group was treated with Guhong injection combined with conventional treatment. There are no limitations on dosage, frequency, and duration. Additionally, we will exclude studies that combined with other herbal formulas, acupuncture, or moxibustion. 4.OutcomesThe primary outcomes will include the frequency, severity, and duration of angina pectoris attacks, electrocardiogram changes, and dose of nitroglycerin. The secondary outcomes will include blood lipid profile (triglyceride, total cholesterol, low-density lipoprotein cholesterol, and high-density lipoprotein cholesterol), Traditional Chinese Medicine syndrome score scale, and adverse events.

Information sources: (1) electronic databases: EMBASE, PubMed, Cochrane Library, Web of Science, China National Knowledgelnfrastructure (CNKI), WanFang Database, Chinese Biomedical Database (CBM), Chinese Scientific Journal Database (VIP). (2) Search for other resources: We will also retrieve grey documents and clinical trial registers, such as WHO International Clinical Trials Registry Platform (ICTRP), the Chinese Clinical Trial Register (Chi CTR) and the Clinical Trials, to supplement the electronic databases. Google Scholar and Baidu Academic will be involved to search relevant literature. We will also manually retrieve relevant literature from Clinical Trials Register. In addition, reference lists of eligible studies 
will be performed manually so as to avoid missing vital information.

Main outcome(s): The primary outcomes will include the frequency, severity, and duration of angina pectoris attacks, electrocardiogram changes, and dose of nitroglycerin.

Additional outcome(s): The secondary outcomes will include blood lipid profile (triglyceride, total cholesterol, low-density lipoprotein cholesterol, and high-density lipoprotein cholesterol), Traditional Chinese Medicine syndrome score scale, and adverse events.

Data management: Two researchers will independently extract data according to a standard data extraction form by using Excel2013 software. The details of the form are as follows: basic information of studies (authors, title, and publication year), participants characteristics (age, gender, numbers, and course of disease), interventions, outcome indicators, adverse events, etc. If there is any incomplete information in the study, we will contact the corresponding author. All disagreements will be resolved by consulting a third researcher.

Quality assessment / Risk of bias analysis: The risk of bias will be assessed independently by two researchers according to the tool of Cochrane Collaboration. The assessment items mainly contain random sequence generation, allocation concealment, blinding of participants and personnel, blinding of outcome assessors, incomplete outcome data, selective outcome reporting, and other sources of bias. Each item will be evaluated into 3 grades: "low risk", "high risk", and "unclear risk". If necessary, a third researcher will be involved to resolve the disagreements.

Strategy of data synthesis: Data synthesis and statistical analysis will be performed using the RevMan $\mathbf{5 . 3}$ software provided by Cochrane Collaboration. The standard mean difference (SMD) or mean difference (MD) with $95 \%$ confidence interval (Cl) will be used to calculate the continuous data, while the dichotomous date will be measured by the rate ratio (RR) or odds ratio (OR) with $95 \% \mathrm{Cl}$. For the assessment of heterogeneity, the Chi2 and 12 test will be carried out. If there is no significant heterogeneity among studies (120.1), we will use a fixed-effect model, but a randomeffects model will be employed if there exists heterogeneity $(12 \geq 50 \%, P<0.1)$. Moreover, subgroup analysis and sensitivity analysis will be conducted to find the potential reasons for heterogeneity.

Subgroup analysis: When there is significant heterogeneity among the studies, subgroup analysis will be performed according to following factors: age, gender, types of CHD, the dosage of Guhong injection, and course of treatment.

Sensitivity analysis: Sensitivity analysis will be conducted by eliminating included studies one by one and changing the statistical methods to assess the stability and reliability of analytical results.

Language: Chinese and English.

Country(ies) involved: China.

Keywords: Coronary heart disease, Traditional Chinese Medicine, Guhong injection.

Dissemination plans: Our results of work will disseminate in professional academic journals.

Contributions of each author:

Author 1 - Sihua Che - The author drafted the manuscript.

Email: 294347339@qq.com

Author 2 - Meiling Wang - The author provided statistical expertise.

Author 3 - Le Liu - The author contributed to the development of the selection criteria, and the risk of bias assessment strategy.

Author 4 - Shumao Zhang - The author read, provided feedback and approved the final manuscript.

Author 5 - Guijun Shi - The author provided funding acquisition and administrated project. 